\title{
Post-intensive care syndrome: impact, prevention, and management
}

Cite as: Colbenson GA, Johnson A, Wilson ME. Post-intensive care syndrome: impact, prevention, and management. Breathe 2019; 15: 98-101.

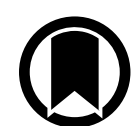

CrossMark
Millions of people worldwide have survived an admission to the intensive care unit (ICU), and the number of survivors is growing [1]. While these patients have survived a life-threatening illness, most survivors suffer important long-term complications [2]. Post-intensive care syndrome (PICS) is a term that describes the cognitive, psychological, physical and other consequences that plague ICU survivors [3, 4]. Our aim is to discuss the prevalence, risk factors, impact, prevention and management of PICS.

\section{Prevalence and risk factors}

Cognitive impairment occurs in $30-80 \%$ of ICU survivors, it may vary in severity and often lasts for years [3, 5]. Psychiatric illnesses, such as anxiety, depression or post-traumatic stress disorder (PTSD), occur in $8-57 \%$ of patients and also can persist for years [3]. New physical impairment occurs in $25-80 \%$ of patients $[3,6]$. In addition, patients may have new symptoms such as dyspnoea or pain, sexual dysfunction, impaired pulmonary function, and impaired exercise tolerance [7, 8]. The prevalence of post-ICU sequelae varies widely among different patient populations. Some patients survive ICU illness without any significant impairment, but a notable proportion experience impairment in multiple areas [9].

Major risk factors and potential biological mechanisms for post-ICU deficits have previously been outlined [10]. The major risk factor for long-term cognitive impairment is presence of delirium and duration of delirium [5, 11]. Other possible associations include hypoglycaemia, hyperglycaemia, hypoxaemia, hypotension and sedation [10]. Risk factors for post-ICU physical impairment may include a diagnosis of acute respiratory distress syndrome, prolonged mechanical ventilation, sepsis, multiorgan failure, exposure to systemic corticosteroids and others [10]. A higher frequency of post-ICU psychological sequelae has previously been associated with pre-ICU psychiatric symptoms, younger age, female sex, poor recall of the ICU stay and ICU sedation [10].

\section{Impact of PICS}

PICS-related impairments often have a profound impact on patients' and families' lives. Approximately one third of patients do not go back to work, another third of patients do not go back to their pre-ICU job or at the same pre-ICU salary [6]. Some patients cannot afford medications or travel to outpatient follow-up or to pay for in-home physical therapy sessions due to financial constraints or lack of resources. In addition, ICU survivors are at high risk for readmission to the hospital and ICU [12]. One quarter of patients require assistance in activities of daily living a year after ICU admission and this can have a tremendous effect on family members [6]. Approximately half of families providing care have 
to make a major adjustment to their working life, usually limiting the household salary [6]. Some families have to move to a less expensive home, lose their savings, delay medical care for other family members, or delay educational plans [13]. Family members can also develop anxiety, depression and PTSD that can persist beyond the hospitalisation, termed PICS-family [14].

\section{Prevention of PICS}

Several interventions have been found to prevent the development of psychological, cognitive, and physical consequences of ICU hospitalisation. The American College of Critical Care Medicine and the Society of Critical Care Medicine updated the Clinical Practice Guidelines for the Management of Pain, Agitation, and Delirium in Adult patients in the Intensive Care Unit (ICU PAD guidelines) in 2018 [15]. The focus of these guidelines has been adapted into the "ABCDEF" bundle, an evidencebased guide to direct treatment to prevent longterm cognitive impairment, delirium and physical decline in the ICU [16]. This refers to A) assess and manage pain, B) breathing trials and spontaneous awakening, C) choice of sedative, D) daily delirium monitoring, E) early mobility, and F) family engagement and empowerment [16]. Because delirium is associated with mortality in adult ICU patients and is a significant risk factor for cognitive impairment after admission, a significant proportion of the ICU PAD guidelines emphasis is on prevention and treatment of delirium $[15,16]$. The guidelines also highlight daily interruption of sedatives for awakening trials. Daily awakening trials, as compared to awakening trials at provider discretion, have been shown to shorten mechanical ventilation by more than 2 days and length of stay in the ICU by 3.5 days [17]. Sedation should be with nonbenzodiazepine medications and level of sedation should be frequently assessed objectively [15]. Early mobilisation also reduces physical decline, loss of muscle mass, and decreases the duration of delirium [18]. As such, ICUs are employing early mobilisation and integration of physical therapists and occupational therapists into the ICU setting. Encouragingly, use of the "ABCDEF" bundle has led to a three-fold increase in patients returning to independent functioning after hospital discharge in some populations [2].

ICU diaries, where ICU survivors can read what family members and ICU staff wrote about the patient's ICU experiences, have consistently been shown to reduce PTSD $[8,19,20]$.

\section{Post-ICU clinics}

While the majority of ICU patients experience one or more post-ICU impairments, these impairments often go undiagnosed and are inadequately treated. Several possible reasons for inadequate diagnosis and treatment are outlined in table 1.

Post-ICU clinics, first started in 1985 in the UK, were designed to evaluate and treat ICU survivors [21]. The objectives of post-ICU clinics include measurement of post-ICU health including assessments such as a 6-min walk test (6MWT) and/or spirometry to aid in the diagnosis of

Table 1 Possible contributors to inadequate diagnosis and treatment of PICS impairments in the outpatient setting

1) Lack of routine post-hospital follow-up with ICU physicians [2, 21].

2) ICU discharge planning and documentation often focuses on organ-specific issues and may not outline functional impairments that require follow-up [4].

3) Significant responsibility is placed on primary care physicians and outpatient physicians who are often unaware of the intensity of the traumatic and life-threatening experiences that ICU patients may have faced [4].

4) Generalised lack of awareness that PICS exists and is relatively common among ICU survivors [4].

5) ICU discharge planning and documentation often focuses on organ-specific issues and may not outline functional impairments that require follow-up [4].

6) PICS symptoms may be subtle and not readily apparent to the untrained clinician, and may have an onset many months following the acute hospitalisation.

7) No validated, universally used screening tools currently exist for assessing post-ICU patients for PICS [4].

8) No established rehabilitation pathway, as in stroke or traumatic brain injury. Rehabilitation clinicians may have less education on critical care issues that require intervention [4].

9) Lack of established best practice guidelines on how to best treat and support patient survivors [4].

10) Limited access to acute inpatient rehabilitation due to insurance constraints, which often require a minimum of $15 \mathrm{~h}$ of rehabilitation services per week and specific diagnosis codes [4]. 
post-ICU impairments, symptom management, medication reconciliation, discussion of prognosis, and implementation of physical, psychological and cognitive rehabilitation [21]. While the composition and size of each post-ICU clinic is different, most have a team-based approach and may include physicians, nurse practitioners, social workers, physical therapists, occupational therapists, nurses, pharmacists and psychologists. While $-30 \%$ of ICU patients in the UK currently undergo dedicated outpatient follow-up 2-3 months after ICU discharge [21], many other countries have only recently adopted the post-ICU clinic model and have much lower rates of dedicated follow-up. Unfortunately, post-ICU clinics have not been found to improve health-related quality of life more than the standard of care [8]. It can be difficult to predict which patients will benefit from specific ICUcentred follow-up, but it has been suggested that dedicated follow-up should be offered to patients that were on mechanical ventilation for more than 2 days between six and 12 weeks after hospital discharge [22].

\section{Post-ICU rehabilitation}

Rehabilitation efforts with physical and occupational therapists during ICU admission and shortly after represents an important strategy to improve patient outcomes. Rehabilitation efforts should be focused at all three domains of PICS: psychological, physical and cognitive [23, 24]. Regarding physical rehabilitation, outpatient programmes vary, but typically span 6-12 weeks after discharge and can include patient-directed exercises, in-home therapist sessions, telehealth delivery of therapy and can be bundled with cognitive rehabilitation [4, 23, 24]. In one small study, patient-directed rehabilitation exercises showed improvement in 6MWT and anxiety and depression scores [4]. Another study demonstrated improved independent activities of daily living in an intervention group undergoing a 12-week outpatient rehabilitation programme, but strength, as measured by the Timed Up and Go test, was not statistically different between the intervention and control groups [24]. There has been limited, but promising data for post-ICU cognitive rehabilitation [23]. In one randomised control trial, a 12-week cognitive and physical rehabilitation programme improved patients' cognitive executive functioning at 3 months [23]. Standardised cognitive rehabilitation protocols, such as the Goal Management Training protocol, have been developed to guide patients' rehabilitation [24]. Currently, it is recommended that patients with cognitive dysfunction, as defined by a Mini-Mental State Examination score $<24$, receive ongoing assessment of their cognitive status 3-6 months following discharge [23]. Regarding psychological rehabilitation, there is not a standardised approach. However, telephonebased techniques are an efficient and feasible method to counsel patients following discharge and have shown promise in improving patients' and caregivers' ability to cope [25].

\section{ICU survivor peer support groups}

There are a number of models for peer support groups including: community-based groups, psychologist led sessions, peer support meetings within follow-up ICU clinics, patient mentormentee relationships and telemedicine, among others [26]. One of the hurdles that prevent patients from seeking out or attending these groups is their inability to identify their deficits as related to their ICU admission [26]. It is likely that increased awareness of PICS among patients early in the discharge process and early introduction of the concept of peer support groups would help facilitate patients' recognition of their post-ICU deficits and encourage them to seek support.

\section{Conclusion}

In conclusion, although mortality of ICU patients has declined significantly in recent decades, morbidity after admission remains a significant issue. Certain preventative measures during ICU admission and rehabilitation efforts after admission show promise in reducing cognitive and physical consequences in ICU survivors. Post-ICU clinics have the potential to diagnose and treat deficits from ICU illness early, but at this time their efficacy has not been shown to be significantly better than the standard of care. Awareness of how to identify and manage PICS deficits is sorely needed among the clinicians who evaluate ICU survivors. More work is needed to standardise follow-up approaches and emphasis on prevention of cognitive, physical and psychological sequelae from the ICU is essential. 


\section{Conflict of interest}

G.A. Colbenson has nothing to disclose. A. Johnson is co-chair of SCCMs Thrive Peer Support Collaborative. M.E. Wilson has nothing to disclose.

\section{References}

1. Wunsch H, Angus DC, Harrison DA, et al. Variation in critical care services across North America and Western Europe. Crit Care Med 2008; 36: 2787-2793.

2. Modrykamien AM. The ICU follow-up clinic: a new paradigm for intensivists. Respir Care 2012; 57: 764-772.

3. Harvey MA, Davidson JE. Post-intensive care syndrome: right care, right now...and later. Crit Care Med 2016; 44: 381-385.

4. Needham DM, Davidson J, Cohen $\mathrm{H}$, et al. Improving long-term outcomes after discharge from intensive care unit: report from a stakeholders' conference. Crit Care Med 2012; 40: 502-509.

5. Pandharipande PP, Girard TD, Jackson JC, et al. Long-term cognitive impairment after critical illness. N EnglJ Med 2013; 369: 1306-1316.

6. Griffiths J, Hatch RA, Bishop J, et al. An exploration of social and economic outcome and associated health-related quality of life after critical illness in general intensive care unit survivors: a 12-month follow-up study. Crit Care 2013; 17: R100.

7. Herridge MS, Cheung AM, Tansey CM, et al. One-year outcomes in survivors of the acute respiratory distress syndrome. NEngl J Med 2003; 348: 683-693.

8. Mehlhorn J, Freytag A, Schmidt K, et al. Rehabilitation interventions for post-intensive care syndrome: a systematic review. Crit Care Med 2014; 42: 1263-1271.

9. Bruck E, Schandl A, Bottai M, et al. The impact of sepsis, delirium, and psychological distress on self-rated cognitive function in ICU survivors-a prospective cohort study. J Intensive Care 2018; 6: 2

10. Desai SV, Law TJ, Needham DM. Long-term complications of critical care. Crit Care Med 2011; 39: 371-379.

11. Sakusic A, O’Horo JC, Dziadzko M, et al. Potentially modifiable risk factors for long-term cognitive impairment after critical illness: a systematic review. Mayo Clin Proc 2018; 93: 68-82

12. Hua M, Gong MN, Brady J, et al. Early and late unplanned rehospitalizations for survivors of critical illness. Crit Care Med 2015; 43: 430-438.

13. Swoboda SM, Lipsett PA. Impact of a prolonged surgical critical illness on patients' families. Am J Crit Care 2002; 11 459-466.

14. Paparrigopoulos T, Melissaki A, Efthymiou A, et al. Short-term psychological impact on family members of intensive care unit patients. J Psychosom Res 2006; 61: 719-722.
15. Devlin JW, Skrobik Y, Gelinas C, et al. Clinical practice guidelines for the prevention and management of pain, agitation/sedation, delirium, immobility, and sleep disruption in adult patients in the ICU. Crit Care Med 2018; 46: e825-e873.

16. Marra A, Ely EW, Pandharipande PP, et al. The ABCDEF bundle in critical care. Crit Care Clin 2017; 33: 225-243.

17. Kress JP, Pohlman AS, O'Connor MF, et al. Daily interruption of sedative infusions in critically ill patients undergoing mechanical ventilation. N Engl J Med 2000; 342: 1471-1477.

18. Kress JP, Hall JB. ICU-acquired weakness and recovery from critical illness. N Engl J Med 2014; 371: 287-288.

19. Ullman AJ, Aitken LM, Rattray J, et al. Intensive care diaries to promote recovery for patients and families after critical illness: a Cochrane systematic review. Int J Nurs Stud 2015; 52: 1243-1253.

20. Jones C, Backman C, Capuzzo M, et al. Intensive care diaries reduce new onset post traumatic stress disorder following critical illness: a randomised, controlled trial. Crit Care 2010; 14: R168

21. Teixeira C, Rosa RG. Post-intensive care outpatient clinic: is it feasible and effective? A literature review. Rev Bras Ter Intensiva 2018; 30: 98-111.

22. Van Der Schaaf M, Bakhshi-Raiez F, Van Der Steen M, et al. Recommendations for intensive care follow-up clinics; report from a survey and conference of Dutch intensive cares. Minerva Anestesiol 2015; 81: 135-144.

23. Denehy L, Elliott D. Strategies for post ICU rehabilitation. Curr Opin Crit Care 2012; 18: 503-508.

24. Jackson JC, Ely EW, Morey MC, et al. Cognitive and physical rehabilitation of intensive care unit survivors: results of the RETURN randomized controlled pilot investigation. Crit Care Med 2012; 40: 1088-1097.

25. Cox CE, Porter LS, Hough CL, et al. Development and preliminary evaluation of a telephone-based coping skills training intervention for survivors of acute lung injury and their informal caregivers. Intensive Care Med 2012; 38: 1289-1297.

26. McPeake J, Hirshberg EL, Christie LM, et al. Models of peer support to remediate post-intensive care syndrome: a report developed by the Society of Critical Care Medicine Thrive International Peer Support Collaborative. Crit Care Med 2019; 47: e21-e27. 